\title{
ILCEA
}

Revue de l'Institut des langues et cultures

d'Europe, Amérique, Afrique, Asie et Australie

$30 \mid 2018$

Création culturelle et territoires : de l'histoire au

mythe, du réel à l'utopie

\section{Völkische Science Fiction: Deutsche Zukunftsromane mit völkischen Tendenzen (1890- 1933)}

La science-fiction " völkisch » : science fiction allemande à tendance

«nationale-identitaire » (1890-1933)

Völkisch Science Fiction: German Science Fictions Novels with Völkisch

Tendencies (1890-1933)

\section{Maurice Schuhmann}

\section{OpenEdition}

Journals

Édition électronique

URL : http://journals.openedition.org/ilcea/4616

DOI : 10.4000/ilcea.4616

ISSN : 2101-0609

Éditeur

UGA Éditions/Université Grenoble Alpes

Édition imprimée

ISBN : 978-2-37747-033-4

ISSN : $1639-6073$

\section{Référence électronique}

Maurice Schuhmann, « Völkische Science Fiction: Deutsche Zukunftsromane mit völkischen

Tendenzen (1890-1933) », ILCEA [Online], 30 | 2018, Online erschienen am: 31 Januar 2018, abgerufen am 30 April 2019. URL : http://journals.openedition.org/ilcea/4616 ; DOI : 10.4000/ilcea.4616

Ce document a été généré automatiquement le 30 avril 2019

(C) ILCEA 


\title{
Völkische Science Fiction: Deutsche Zukunftsromane mit völkischen Tendenzen (1890-1933)
}

\author{
La science-fiction « völkisch » : science fiction allemande à tendance \\ «nationale-identitaire » (1890-1933) \\ Völkisch Science Fiction: German Science Fictions Novels with Völkisch \\ Tendencies (1890-1933)
}

Maurice Schuhmann

1 Der Machtergreifung der Nationalsozialisten im Jahr 1933 und der Konsolidierung ihrer Macht in Deutschland gingen knapp fünfzig Jahre Wirken und Propagandaaktivität der völkischen Bewegung voraus. Sie bereiteten ihnen den Weg und stellten kulturelle Hegemonie her, die der Nationalsozialismus für seine Wirkungsentfaltung benötigte.

2 Ein wichtiges Medium der Verbreitung völkischen Denkens stellte neben einschlägigen Zeitschriften und Vorträgen seitens ihrer Protagonisten vor allem die Literatur dar. Esoterische und pseudowissenschaftliche Werke, die krude Rassentheorien aufstellten oder das Germanentum glorifizierten, bildeten den einen Strang der Literaturproduktion; einen anderen stellte die Belletristik, besonders die Trivialliteratur, dar. Im Kontext der Belletristik nahm die phantastische Literatur eine Sonderstellung ein. Zur phantastischen Literatur können dabei sowohl Märchen, Legenden und Sagen als auch Zukunftsromane gerechnet werden. Sie bot mit ihrer Affinität zu Mythen und ihrer Nähe zu Utopien eine Reihe von Anknüpfungspunkten für die völkische Ideologie. Dennoch ist dieses Forschungsfeld noch weitgehend unbekannt. Jost Hermand schrieb diesbezüglich in seiner Studie Der alte Traum vom neuen Reich:

Seltsamerweise sind diese (die völkischen) Visionen, Utopien und Zukunftsromane bei der Erforschung der Vorgeschichte des Nationalsozialismus bisher weitgehend übersehen worden, obwohl sie bei der Entstehung und Entfaltung eines auch die breiten Massen ergreifenden Nationalbewusstseins sicher eine beachtliche Rolle haben und für viele jener chauvinistischen Sehnsüchte mitverantwortlich sind, die in den Jahren nach 1933 zu wahnwitzigen, ja bestialisch Überspitzungen der älteren 
deutschnationalen Vorstellungen führte. Die Fülle dieser Schriften ist, sofern man auch die reiche Science-Fiction-Literatur mitberücksichtigt, kaum zu überschauen. So sind allein in den fünfzig Jahren zwischen 1895 und 1945, als dieser Wandlungsprozess ins Chauvinistische und Imperialistische seinen Höhepunkt erlebte, etwa zwei- bis dreihundert solche Romane erschienen, die zum Teil hohe Auflagen erlebten und von Hunderttausenden, wenn nicht Millionen gelesen wurden. (1988: 14)

Im Folgenden möchte ich mich auf die Gattung Science Fiction als eine Teilgattung des utopie-affinen Zukunftsromans konzentrieren. In der grundlegenden Literatur zur deutschsprachigen Science Fiction-Literatur wird diese je nach Standpunkt entweder als "legitimes Erbe des utopischen Romans“ (Schwonke, 1957) oder als „pervertiertes Erbe der Utopie“ (Nagl, 1972) betrachtet ${ }^{1}$.

4 In Deutschland kam die Science Fiction um 1890 auf. Sie trat literarisch z. T. das Erbe der Kolonialromane an (Franke, 1985: 285 ff.). Während im Kolonialroman die Abenteuer noch in fremden Ländern mit fremdartigen Kulturen spielten, verlagerte sich nun der Plot meistens in den Weltraum und auf andere Planeten. Die Science Fiction-Literatur bot viele Anknüpfungspunkte für völkische Vorstellungen und Politikansätze, so dass sich Teile der frühen Science Fiction-Literatur unter dem Label „völkische Science Fiction“ subsummieren lassen.

Bezüglich „völkischer Science Fiction“ orientiere ich mich an der Definition von Michael Novian, der diese wie folgt umschrieb:

Die Terminologie völkische Science Fiction soll demnach lediglich auf literarische Erzeugnisse einer bestimmten abgegrenzten Zeit verweisen, die von Autoren geschrieben wurden, die unter dem Einfluss der völkischen Bewegung standen und aus diesem Eindruck heraus ihre Werke konzipierten. (2013: 69)

6 Sowohl auf die Affinität der frühen Science Fiction-Literatur, die sich ihrer Auflagenhöhe nach großer Beliebtheit in der deutschen Öffentlichkeit erfreute ${ }^{2}$, zur völkischen Bewegung (Nagl, 1972: 89 f.) als auch auf die Bedeutung für die Verbreitung völkischen Denkens (Hermand, 1988: 14) wurde zwar schon in der einschlägigen Forschungsliteratur hingewiesen, aber dennoch bietet jenes Feld noch viele unbekannte Facetten ${ }^{3}$. Dies verwundert vor dem Hintergrund, dass es auch in der jüngeren Vergangenheit wiederholt zu Publikationen rechtsradikaler Science Fiction-Romane in Deutschland kam, die für einen entsprechenden Presserummel sorgten ${ }^{4}$.

7 Im Kontext dessen möchte ich im Folgenden an Hand dreier Beispiele aus dem Dunstkreis der völkischen Bewegung die Frage nach der Affinität sowie die Anknüpfungspunkte von Science Fiction-Literatur und völkischer Ideologie aufzeigen und beleuchten.

Als Beispiele für meine Darstellung habe ich folgende Romane gewählt:

Max Haushofer: Planetenfeuer (1899) ${ }^{5}$

Beowulf (= Maximilian Kern): Der deutsch-englische Krieg. Vision eines Seefahrers (1906)

Friedrich Freksa: Druso oder: Die gestohlene Menschenwelt (1931).

9 Die Romane stechen aus unterschiedlichen Gründen aus der Masse der völkischen Science Fiction-Literatur hervor. Ersterer - Planetenfeuer - gewinnt seine Relevanz dadurch, dass er noch 2010 als Vorlage für ein Radiohörspiel des Bayrischen Rundfunks diente ${ }^{6}$ und sich eine Neuauflage des Romans seitens des Münchner belleville-Verlages in Arbeit befindet ${ }^{7}$. Der unter Pseudonym publizierte Roman Der deutsch-englische Krieg besitzt in Hinblick auf seinen Verfasser Maximilian Kern, dessen Jugendromane bis heute neuaufgelegt werden, Bedeutung. Der an letzter Stelle genannte Roman ist wegen seiner Verbreitung über den deutschsprachigen Raum hinaus von Interesse. Es erschienen Übersetzungen ins 
Englische (1934) ${ }^{8}$ und ins Französische (1960) ${ }^{9}$. Dieser Roman wurde damit auch außerhalb Deutschlands rezipiert.

Die gewählten Beispiele weisen somit über den engen zeitlichen und örtlichen Kontext hinaus und bieten z.T. noch für aktuelle literaturwissenschaftliche Debatten Anknüpfungspunkte, während die meisten Romane jenes Genres mittlerweile in Vergessenheit geraten sind.

Darüber hinaus decken die drei Romane unterschiedliche Perioden und Facetten der völkischen Science Fiction-Literatur ab. Haushofers Roman und Der deutsch-englische Krieg entstanden im Deutschen Kaiserreich, während Druso vor dem Erfahrungshintergrund der Weimarer Republik verfasst wurde. Es sind daher Beispiele für Romane, die einen Anteil hatten an der ideologischen Mobilmachung und Implementierung völkischen Denkens von der Entstehung jener Bewegung bis $\mathrm{zu}$ ihrem Höhepunkt in Form der Machtergreifung durch die NSDAP.

Weiterhin lassen sie sich in den folgenden Unterkategorien einordnen, die die Bandbreite völkischer Science Fiction-Literatur weitgehend abdecken:

Weltuntergangsszenario (Planetenfeuer)

Kriegsszenario (Der deutsch-englische Krieg)

Erlösungs- und Befreiungsszenario (Druso)

Die zeitgenössische Rezeption jener Literatur lässt sich heute leider nicht mehr rekonstruieren. Eine direkte Auseinandersetzung mit jenen Romanen in Feuilletons ist ebenso wenig vorhanden wie eine Analyse der Leserschaft jener Literaturgattung in dieser Zeit. Es lassen sich daher lediglich generelle Mutmaßungen über die Bedeutung anstellen.

Bevor ich die drei Romane beleuchte, gilt es noch den Begriff des „Völkischen“ näher zu bestimmen. Ursprünglich wurde das Adjektiv „völkisch“, das 1909 erstmalig in ein deutsches Lexikon Eingang fand (Meyer's, 1909: 229), lediglich als Eindeutschung des Begriffes „national“ durch rechte/rechtsextreme Gruppierungen im Deutschen Kaiserreich definiert. In neueren Nachschlagewerken wie dem Dtv-Brockhaus-Lexikon von 1986 wird der Begriff folgendermaßen gefasst:

Etwa 1875 aufgekommene Verdeutschung des Wortes „national“, bes. i.S. eines auf

dem Rassegedanken begründeten und daher entschiedenen antisemit.

Nationalismus verwendet. (1986: 214)

Diese Definition werde ich im Weiteren - neben der von „völkischer Science Fiction“als Grundlage meiner Darstellung nutzen.

\section{Völkische Weltuntergangsszenarien - Max Haushofers Planetenfeuer}

Der Autor Max Haushofer (1840-1907) ist mittlerweile weitgehend in Vergessenheit geraten. Er war ein deutscher Philosoph, Nationalökonom, Politiker (als Abgeordneter der Stadt München für die Vereinigte Liberale im bayrischen Landtag) und Autor sowie Mitbegründer des im Kontext des Freidenkertums stehenden Monistenbundes in Deutschland $^{10}$. Die Lehre des Monismus als solche mit ihren sozialdarwinistischen Tendenzen weist Überschneidungen zum völkischen Denken jener Zeit auf. Politisch ist Haushofer allerdings eher im nationalliberalen Lager zu verorten. 
16 Neben dem Science Fiction-Roman Planetenfeuer hat er $u$.a. in der populärwissenschaftlichen Schrift An des Daseins Grenzen. Geschichten und Phantasien (1908) seine Zukunftsdiagnosen dargelegt, die vereinzelt utopische Elemente beinhalten. Der Roman Planetenfeuer wurde im Rahmen seines Gesamtwerkes relativ wenig rezipiert ${ }^{11}$, obwohl er für die Entwicklung des Genres der Science Fiction-Literatur in Deutschland einen Meilenstein darstellt.

Nach Auffassung von Robert Hahn hat Haushofer zwar kein direkt völkisches Gedankengut propagiert, aber solches, das sich gut in den Diskurs einpasste (Hahn, 2003: 36). Manfred Nagl verortete ihn in seiner maßgeblichen Studie über die Anfänge deutschsprachiger Science Fiction-Literatur eindeutig im völkischen Diskurs (Nagl, 1972: 96). Nach Angaben von Franz Wegener befand sich aber auch eine Kopie des Romans im persönlichen Besitz von Reichsführer SS Heinrich Himmler (Wegener, 2001: 103).

Haushofer beschreibt in Planetenfeuer eine wohlgeordnete Gesellschaft im Jahre 1999, die in einer Art Staatssozialismus und in einem nunmehr fünfzig Jahre anhaltenden Frieden lebt. Das Konzept des Staatssozialismus wird von ihm dabei aus nationalliberaler Sicht durchaus kritisch kommentiert. Inwieweit Johann Gottlieb Fichtes Geschlossener Handelsstaat (1800) Pate für die Sozialismusvorstellung gestanden hat, lässt sich nicht sagen. Hierfür sind die Angaben zum sozialen System zu ungenau. Der politische Konflikt zwischen dem zeitgenössischen Sozialismus und liberalen Positionen wird bei Haushofer zu einem literarischen Motiv. Ein Merkmal der sozialistischen Gesellschaft ist - ganz im Sinne Friedrich Nietzsches - die Dekadenz. Der Begriff der Dekadenz, der auch in völkischen Kreisen redundant kritisiert wurde und später in der Philosophie Oswald Spenglers eine wichtige Rolle einnimmt, ist in jener Strömung der Science Fiction eng mit dem Untergang von Gesellschaften verknüpft.

Die im Roman dargestellte Welt steht allerdings kurz vor der Vernichtung durch den Zusammenprall mit einem anderen Planeten. Der Untergang der Erde wird aber nur von einer kleinen Anzahl von Menschen, einer Elite, vorhergesehen, während die Masse weiter ihrem Alltag nachgeht. Hier zeigt sich wieder eine Gegenüberstellung der die Gefahr verkennenden Masse und der erkennenden Minderheit einer Elite. Diese Masse gleicht in ihrer Haltung dem Bild von Nietzsches „letzten Menschen“, wie er es als Gegenteil des Übermenschen im Zarathustra zeichnet. Sie sind wie diese auch dem Untergang geweiht.

Den Aufprall des Planten überlebt eine Anzahl von Menschen, die nach diesem Inferno die Welt neu aufbauen. Die Apokalypse hat somit wiederum eine reinigende Wirkung.

21 Die von Nietzsches Philosophie beeinflussten Facetten der Handlung sind unverkennbar. Haushofer bedient sich ihrer recht offen. Es sind auch jene Facetten - wie etwa die Gleichsetzung einer sozialistisch organisierten Gesellschaft mit der Dekadenz - die ihn anschlussfähig an das völkische Denken machen. Daneben klingen aber auch wiederholt patriotische Töne an, die seine eigene politische Verortung untermalen.

Jost Hermand greift in seiner Analyse der dystopischen Zukunftsromane Planetenfeuer heraus und erklärt, dass dieser sich

[...] in Form einer apokalyptischen Vision gegen jene nationalliberalen (sic!), feministischen und sozialdemokratischen Tendenzen innerhalb des wilhelminischen Reiches wendet, die nach Meinung des Autors zu einer fortschreitenden Verkrüppelung aller menschlichen Urinstinkte führen müssen [... ]. Die gesellschaftliche Perspektive geht dabei eindeutig von den Oberschichten, den Edelmenschen und Volksführern aus. Sie sind die wahrhaft "national“ 
Denkenden, während das Volk - wie Nietzsches „letzte Menschen“ - in einem dumpfen Materialismus vor sich hin vegetiert. (Hermand, 1988: $57 \mathrm{f}$.) Werke auch nach 1945 noch neuaufgelegt wurden - z. B. der erstmals 1909 erschienene Roman In der Wildnis des Gran Chaco (zuletzt: 2012) oder der 1910 publizierte Band Das Erbe des Pharao (zuletzt: 1955). Neben diesen Werken verfasste er unter dem Pseudonym Beowulf 1906 auch den Science Fiction-Roman Der deutsch-englische Krieg. Vision eines Seefahrers, der nach Angaben von Franke mindestens in drei Auflagen erschien und eine Gesamtauflage von 7000 Exemplaren erreichte (Franke, 1985: 331). Der Verlag Hermann Walther, bei dem der Roman erschien, publizierte schwerpunktmäßig militaristische und rechtskonservative Titel. Der Roman an sich steht in der Tradition des politischmilitärischen Zukunftsromans, der sich zwischen dem ausgehenden 19. Jahrhundert und dem Ausbruch des I. Weltkrieges in Deutschland großer Beliebtheit erfreute, und zeigt Parallelen zum stilbildenden Roman 1906 von Ferdinand Grautoff (Pseudonym: Seestern) auf. Er wurde durch antidemokratische Erlebnisberichte und Erzählungen über den I. Weltkrieg später verdrängt.

Die Wahl des Pseudonyms Beowulf wurde von Franke wie folgt erklärt:

[D]as Pseudonym „Beowulf“ soll eine Erzählung über seefahrende Kriegshelden ankündigen, wobei Autor und Leser wohl kaum den Ort der Geburt Beowulfs und die Schauplätze seiner Taten assoziieren, die sämtlich in Skandinavien liegen, sondern vielmehr das Land, in dem das Beowulf-Epos niedergeschrieben wurde: England. (Franke, 1985: 78 f.)

Die Geschichte von Der deutsch-englische Krieg spielt im 20. Jahrhundert - zu einem nicht näher definierten Zeitpunkt. Um 19xx kommt es zu einem „allgemeinen Negeraufstand“ (Beowulf, 1906: 3) in Südafrika, für den die Engländer die Deutschen verantwortlich machen. Die Folge ist, dass England Deutschland den Krieg erklärt, der aber mit einem fairen Friedensschluss endet. Nach Aussage des Erzählers im letzten Satz des Romans benötigt Deutschland den Frieden: „Von einem solchen hängt es ab, ob die germanische Rasse dermaleinst die Welt regieren wird oder nicht.“ (Beowulf, 1906: 119) 
Dem Krieg hingegen kommt eine reinigende Wirkung zu. Ein Gedanke, der zu jener Zeit en vogue ist und sich auch in ähnlicher Form im Werk des Philosophen Friedrich Nietzsche findet:

Wie von einem reinigenden Gewitter war die dicke Staubschicht von Parteigezänke, Konfessionshader, Klassenhass, Philisterei und falschem Idealismus, die uns in den langen Friedensjahren zu ersticken gedroht hatte, weggefegt, und wie ein blankes Schild leuchtete wieder Vaterlandsliebe und Königstreue weithin auf. (Beowulf, 1906: 134)

Neben diesem Bezug zur „germanischen Rasse“ im Abschluss seines Romans, finden sich im Plot der Geschichte nationale Töne, die die nationalen Eigenschaften der Deutschen herausstellen und glorifizieren:

Dieses feinfühlige Unterscheiden zwischen dem feindlichen Staat und seinen Individuen stellt dem Gerechtigkeitsgefühl und Takt unseres Volkes sicherlich ein gutes Zeugnis aus. (Beowulf, 1906: 35)

Der Rassebegriff ist ein in diesem Roman mehrfach auftauchender Gedanke. Der Verfasser warnt u.a. im Nachwort vor „möglichen zukünftigen Racenkämpfe[n]“ (Beowulf, 1906: 122).

31 Nessun Saprà ordnet den Roman in dem Nachschlagewerk Lexikon der deutschsprachigen Science Fiction und Fantasy als nationalistischen und rassistischen Zukunftsroman in der Tradition von Karl Bleibtreu und August Niemann, zwei anderen Autoren aus dem Spektrum der militärisch-politischen Science Fiction, ein (Saprà, 2000: 150). Das literarische Niveau ist vergleichbar mit dem eines Landser-Heftchens. Es handelt sich um einen klassischen Vertreter der Trivialliteratur.

\section{Völkische Erlösungs- und Befreiungsszenarios - Kurt Friedrich Freksas Druso oder: Die gestohlene Menschheit}

Kurt Friedrich Freksa (1882-1955) war zum Zeitpunkt der Veröffentlichung von Druso oder: Die gestohlene Menschheit längst kein Unbekannter mehr in der völkischen Bewegung. Er war zuvor bereits Redakteur bei der völkisch-orientierten Satirezeitschrift Phosphor (1919), die vom Alldeutschen Verband ${ }^{12}$ herausgegeben wurde. Auch nach 1933 blieb er als Autor esoterischer Werke tätig, aber er schrieb keine Science Fiction-Literatur mehr. Druso gilt wie auch der Roman Planetenfeuer als ein "Meilenstein“ der deutschsprachigen Science Fiction-Literatur ${ }^{13}$. Diese Klassifizierung beruht auf den literarischen Qualitäten des Romans.

Schon vor dem Erscheinen von Druso hatte Freksa sich an Science Fiction-Geschichten probiert wie z. B. Unter dem Luftfrosch (1921) und Die Mongolei im Winterschlaf(1928). Letztere wurde fast wortwörtlich in seinen Roman Druso integriert. Diese Texte weisen bereits deutliche völkische Tendenzen auf - und sind mitnichten Antikriegsutopien wie die Veröffentlichung in dem 2007 von Detlef Münch herausgegebenen Sammelband Der Krieg der Zukunft vor 100 Jahren. Antikriegsutopien als Interpretation suggeriert.

In seiner Kurzgeschichte Unter dem Luftfrosch schreibt er - mit dem damaligen völkischen Denken konform gehend:

Sie [Alfred Bärwald und seine Frau] fragten beide nichts danach, dass Deutschland als politische Einheit aufgehört hatte zu existieren und der Ausbeutung durch die 
fremden Völker, die das Land unter ihre Kontrolle nahmen, verfiel. (Freksa, 1921: 43)

Die Handlung des Romans Druso beginnt im 20.Jahrhundert, als sich der Atlantier (Bewohner von Atlantis) Alf Bentinks, der gleichzeitig der Ich-Erzähler der Geschichte ist, einfrieren lässt. Ein paar Jahrhunderte später werden er und einige seine Gefährten wieder aufgeweckt - und sie sehen sich mit einer völlig veränderten Welt konfrontiert. Die Welt ist - bis auf eine kleine Gemeinschaft von Nordländern und einzelnen versprengten (höheren) Menschen - von den Bewohnern des Raubplaneten Druso unterjocht. Die Bewohner jenes Raubsterns beuten sowohl die Arbeit der jungen Männer als auch die Frauen aus. Erst unter der Führung von Alf Bentinks gelingt es, die Eindringlinge zu verjagen und die Menschen zu befreien. Der Roman endet mit den programmatischen Worten:

Dennoch bleibt der Krieg der Vater allen Geschehens! Niemals hört der Kampf auf, solange Welten bestehen. [...] Werdet als die Beherrscher der Erde nicht wie die Drusonen selbst schwächlich und selbstsüchtig! (Freksa, 1931: 298 f.)

Die Anknüpfungspunkte und Aspekte des völkischen Denkens sind dabei unübersehbar - Antikommunismus, Rassismus und Züchtungsgedanke. Sie tauchen bereits in der Beschreibung der Gesellschaft des 20. Jahrhunderts auf.

Das sowjetische Wirtschaftssystem ist bereits gescheitert (Freksa, 1931: 9), die Menschheit bzw. die „weiße Rasse“ wird durch die „gelbe Rasse“ bedroht (ebd.: 10) und die Menschenrasse wird durch stetige Selektion bei der Geburt hochgezüchtet (ebd.: 23). Nicht lebenswerte Säuglinge werden „thermisch vernichtet“" (ebd.). Freksa bedient sich damit sowohl der Theorie des Rassenkampfes und des Sozialdarwinismus, der in der Euthanasie zum Vorschein kommt, als auch des Antikommunismus als drei wesentlichen Kernelementen völkischen Denkens.

Unter Umständen lässt sich in diesem Kontext auch der - in der Science Fiction-Literatur zu dieser Zeit verbreitete - Rückgriff auf den Atlantis-Mythos ideologisch erklären und interpretieren. Atlantis galt in den esoterisch-okkulten Schriften häufig als Heimat der Arier bzw. nach Guido von List, einem der okkulten Ideengeber des Nationalsozialismus, als die Heimat der Atlantier, aus denen sich die Arier als Rasse entwickelt haben. Der Atlantismythos findet sich auch in anderen Werken der völkischen Science FictionLiteratur wieder - z. B. in den Romanen von Edmund Kiss, der später zum persönlichen Stab Heinrich Himmlers gehörte ${ }^{14}$.

39 Neben dem mythischen Bezug auf Atlantis bedient er sich eines anderen Mythos - dem der Kyffhäusersage. So wie einst König Barbarossa aus seinem Schlaf erwachen, seinem bedrohten Volk zu Hilfe eilen und ein neues Reich begründen wird, so tut es ebenfalls der Held dieses Romans. Auch dieser Mythos wurde vom Nationalsozialismus vereinnahmt und bildete einen wichtigen Bezugspunkt für die nationale Bewegung vor 1933.

Wie in vielen Romanen jenes Genres taucht auch in Druso eine Wunderwaffe auf, die es dem Helden ermöglicht, sein Volk zu befreien. Die Idee von Wunderwaffen, die später von der nationalsozialistischen Propaganda für Durchhalteparolen instrumentalisiert wurde, ist ein redundantes Thema - vor allem in der politischen-militärischen Science FictionLiteratur jener Zeit.

4 Der Höhepunkt der Antizipation des Nationalsozialismus findet sich in der Beschreibung der Bewohner des Raubsterns, der Drusen. Diese sind - wie Manfred Nagl in seiner Studie Science Fiction in Deutschland bereits zeigte - mit klassischen völkischen Stereotypen des 
"Juden“ beschrieben (Nagl, 1972: 168). Sie sind als Parasiten dargestellt, die von der fremden Arbeit leben und nur durch die Ausbeutung anderer Kulturen existieren können. Freksa lässt einen Bewohner der Erde über die Eindringlinge sagen:

Darum brauchen sie die Menschen, weil sie degeneriert sind. Sie brauchen die Milch der Frauen, sie brauchen die Wärme der Frauen, sie brauchen die Arbeitskraft unserer jungen Männer. (1931: 257)

Die von Nagel präsentierte Lesart der Romane Planetenfeuer und Druso - als Beispiele für völkische Science Fiction - ist allerdings sowohl in der Forschung als auch in der Science Fiction-Leserschaft umstritten. Dina Brandt bemerkte hierauf bezogen in ihrer Untersuchung Der deutsche Zukunftsroman:

Nagl ist sich der Fiktionalität dieser Texte nicht bewusst. Ganz offenbar sucht er Beweise, die seinen Ideologieverdacht belegen und daher unterscheidet er nicht mehr zwischen Phantasie und Wirklichkeit. (2007: 27)

Erstaunlich ist, dass der Reckendorf Verlag, bei dem dieser Roman erschien, die Gründung eines deutsch-jüdischen Buchdruckers war. Wie Freksa an diesen Verlag kam und wieso trotz der erkennbaren antisemitischen Züge der Roman dort erschien, lässt sich heute nicht mehr nachvollziehen.

Freksa stellte seinem Roman eine Passage aus Oswald Spenglers Essay Der Mensch und die Technik voran, einer Fortsetzung seines Werkes Der Untergang des Abendlandes. Freksa muss dies nach der Fertigstellung seines Manuskriptes im Juni 1930 nachträglich ergänzt haben, denn Spenglers Pamphlet erschien erstmalig 1931 beim C. H. Beck Verlag. Im Romantext findet sich eine weitere Referenz auf Spengler, einen der geistigen Wegbereiter des Nationalsozialismus, in der Beschreibung der Drusonen. Die Spenglers Gedankengut am deutlichsten reproduzierende Passage ist folgende:

Die Lehre, dass wir es mit einem der Dekadenz entgegengehenden Schmarotzervolk zu tun hätten, das eigene Arbeit kaum noch leisten könnte, schien durch alle Tatsachen bestätigt zu sein. (Freksa, 1931: 191)

Der Begriff Schmarotzervolk stellt eine Analogie zu dem von Spengler benutzen Terminus „Wirtsvolk“ her. In seiner Schrift Der Untergang des Abendlandes hatte dieser die Juden als Fremdkörper in Europa beschrieben und ihnen eine zersetzende Wirkung auf ihre „Wirtsvölker“ zugeschrieben. Auf diese Vorstellung scheint Freksa hier zu rekurrieren.

Peter S. Fisher bezeichnete den Roman als ,ideologically in line with both National Socialism and Weimar's 'Conservative Revolution" (Fisher, 1991: 125). Druso ist damit ein sehr eindeutiges Beispiel für die Verquickung völkischer Ideologiefragmente, utopischer Elemente und Science Fiction-Literatur. Er ist darüber hinaus ein Beispiel dafür, wie die Philosophie jener Bewegung in der Science Fiction-Literatur ihren (vulgären) Ausdruck findet.

\section{Fazit}

Jeder der drei Romane vertritt für sich genommen eine Spielart des völkischen Science Fiction-Romans. Ihnen ist allen ein mehr oder weniger stark ausgeprägter Rassismus und Nationalismus gemeinsam, der im Falle von Maximilian Kern darüber hinaus sehr deutlich chauvinistische Züge trägt. Sowohl bei Maximilian Kern als auch bei Friedrich Freksa taucht Deutschland als Handlungsort namentlich oder durch geographische Beschreibungen auf. Der Krieg spielt in beiden Werken zudem eine zentrale Rolle. Kern lobt ihn als reinigende Kraft, Freksa beschreibt ihn als Akt der Befreiung der Menschheit 
von der Unterjochung. Diese von Kern beschriebene reinigende Wirkung bezieht sich auf eine als dekadent-verkommen beschriebene Gesellschaft, die von allen drei Autoren angesprochen wird - und in der Darstellung Freksas an die Philosophie Oswald Spenglers angelehnt ist. Antisemitismus spielt hingegen nur bei Friedrich Freksa eine deutliche Rolle, der die parasitären Aliens mit klassischen antisemitischen Stereotypen ausmalt. Auch die mythischen Bezüge, die Anknüpfungspunkte für völkisches Denken boten, tauchen nur in Druso auf. Neben dem Atlantis-Mythos finden sich noch weitere, hier nicht näher thematisierte Bezüge zur germanischen Mythologie im Roman.

Die oben genannten Elemente des Rassismus und des Chauvinismus werden vollständig in die jeweilige Handlung integriert und durchziehen jeweils den Plot der Geschichte. So schwingt bei diesen Romanen stets völkische Ideologie mit, die teils eher versteckt wie bei Planetenfeuer, teils plakativ-offen wie bei Druso in Erscheinung tritt.

In die Darstellung der zukünftigen Zeit projiziert Max Haushofer seine Befürchtungen vor einer dem Untergang geweihten, dekadenten Gesellschaft, während Freksa von einer durch eine fremde Macht ausgebeuteten Welt erzählt. Sie warnen damit - ebenso wie Maximilian Kern - vor einer durch Gefahren bestimmten (potentiellen) Zukunft und weisen Züge einer Dystopie auf.

Dagegen werden aber auch einzelne politische Forderungen als verwirklicht und in ihren positiven Auswirkungen beschrieben, d. h. es kommt auch eine positive Utopie zum Tragen. Plastisch wird dies an scheinbar nebensächlichen Beschreibungen wie der der Euthanasie in Druso. Hier wird ein als Fortschritt dargestelltes Vorgehen in seinen positiven Auswirkungen beschrieben und propagiert, was später im Nationalsozialismus zur Realität wurde.

Ein Randaspekt ist weiterhin die Referenznahme auf Nietzsche, der eine konservative und partielle Rezeption $\mathrm{zu}$ Grunde liegt - sei es in Form namentlicher Erwähnung bei Haushofer oder durch indirekte Bezugnahmen wie im Falle von Maximilian Kern, der in seiner Glorifizierung des Krieges wiederholt auf bekannte Aphorismen Nietzsches anspielt. Die Nietzsche-Aphorismen und Gedanken, auf die dabei zurückgegriffen wird, sind auch jene, auf die später der Nationalsozialismus seinen Nietzsche-Kult begründet. Es handelt sich dabei vorrangig um Referenzen auf Also sprach Zarathustra, in dem sich Nietzsche wiederholt positiv auf den „Krieg“ bezieht.

53 Auf dem Wege der literarischen Zukunftsvisionen ließen sich völkische Gedanken und z. T. Schreckensszenarien, die auch von völkischen und nationalsozialistischen Kreisen bedient wurde, bereits einer breiten bzw. breiteren Öffentlichkeit zugänglich machen. Umgekehrt konnte sich der Nationalsozialismus später bei den in dieser Literatur präsenten Motiven für seine Propaganda bedienen. Ein Beispiel hierfür ist die vielbeschworene Wunderwaffe V2, die als solches in der militärisch-geprägten Science Fiction-Literatur jener Epoche ein redundant auftauchendes Thema war. Wegen des hohen Verbreitungsgrades der Science Fiction-Literatur in Deutschland in jenen Jahren, wo sie nach Manfred Nagl zeitweilig die zweitbeliebteste literarische Gattung darstellte, ist von einer starken Verbreitung und weitreichender Rezeption jener Gedanken auszugehen. 


\section{BIBLIOGRAPHIE}

\section{Werke}

BEOWULF (= Maximilian KERN) (1906), Der deutsch-englische Krieg. Vision eines Seefahrers, Berlin: Hermann Walther Verlagsbuchhandlung Berlin.

FREKSA Friedrich (1931), Druso oder: Die gestohlene Menschenwelt, Berlin: Reckendorf.

FREKSA Friedrich (1960), Druso (übersetzt und herausgegeben von G. H. Gallet), Paris: Librairie Hachette.

FREKSA Friedrich (1928 / 2007), „Die Mongolei im Winterschlaf“, D. Münch (dir.), Der Krieg der Zukunft vor 100 Jahren. Antikriegsutopien. Band 3: Die Nachkriegsjahre 1919-1928, Dortmund: Synergen Verlag, 48-56.

FREKSA Friedrich (1921 / 2007): „Unter dem Luftfrosch“, D. Münch (dir.), Der Krieg der Zukunft vor 100 Jahren. Antikriegsutopien. Band 3: Die Nachkriegsjahre 1919-1928, Dortmund: Synergen Verlag, 39-47.

HAUSHOFER Max (1908), An des Daseins Grenzen. Geschichten und Phantasien, München: Beck'sche Verlagsbuchhandlung.

HAUSHOFER Max (1899), Planetenfeuer, Stuttgart: Cotta Verlag Stuttgart.

HAUSHOFER Max \& OTTER Martin (Hrsgg.) (2017), Planetenfeuer (mit einem Essay von Martin Otter), München: Belleville Verlag Michael Farin (im Druck).

KERN Maximilian (1955): Das Erbe des Pharao, Stuttgart: Union Deutsche Verlagsgesellschaft.

KERN Maximilian (2012), In der Wildnis des Gran Chaco, Paderborn: Salzwasser Verlag.

\section{Sekundärliteratur}

BELLEVILLE VeRLAG (Webseite), online unter <www.belleville-verlag.de/scripts/buch.php?ID=505> (20. Juni 2017).

BLoch Robert N. (1984a): „Friedrich Freksa“, J. Körber (Hrsg.), Bibliographisches Lexikon der utopisch-phantastischen Literatur, Meitingen: Corian Verlag.

BLoch Robert N. (1984b): „Max Haushofer“, J. Körber (Hrsg.), Bibliographisches Lexikon der utopischphantastischen Literatur, Meitingen: Corian Verlag.

BRANDT Dina (2007), Der deutsche Zukunftsroman 1918-1945. Gattungstypologie und sozialgeschichtliche Verortung, Tübingen: Niemayer (= zugl. Univ.-Diss. München, 2004).

Dtv-Brockhaus-Lexikon in 20 Bänden (1986), München: Deutscher Taschenbuch Verlag.

FICHTE Johann Gottlieb (1980), Der geschlossene Handelsstaat, Hamburg: Meiner Verlag.

FISHER Peter S. (1991), Fantasy and Politics. Visions of the Future in the Weimar Republic, Madison, London: The University of Wisconsin Press.

FRANKE Henning (1985), Der politisch-militärische Zukunftsroman in Deutschland 1904-1914, Frankfurt, Bern, New York: Peter Lang Verlag. 
HAHN Robert (2003), „Der Erfinder als Erlöser. Führerfiguren im völkischen Zukunftsroman“, H. Hesselborn (Hrsg.), Utopie, Antiutopie und Science Fiction im deutschsprachigen Roman des 20. Jahrhunderts, Würzburg: Könighausen und Neumann, 29-47.

HERMAND Jost (1988), Der alte Traum vom neuen Reich. Völkische Utopien und Nationalsozialismus, Frankfurt am Main: Athenäum Verlag.

INNERHOFER Roland (1996), Deutsche Science Fiction 1870-1914. Rekonstruktion und Analyse der Anfänge einer Gattung, Wien, Köln, Weimar: Böhlau Verlag.

Jehmlich Reimer (1980), „Phantastik - Science Fiction - Utopie. Begriffsgeschichte und Begriffsabgrenzung“, C. W. Thomsen \& J. M. Fischer (Hrsgg.), Phantastik in Literatur und Kunst, Darmstadt: Wissenschaftliche Buchgesellschaft, 11-33.

JOKAN RADIO HÖRSPIELINFO, online unter <www.jokan.de/sf-aktuell.html> (20. Juni 2017).

Meyer's Großes Konversations-Lexikon (1909), Band 6: Veda bis Zz, Leipzig: Bibliographisches Institut.

MÜNCH Detlef (2005), Science Fiction Erzählungen und Kriegsutopien in deutschen Periodika 1880-1949, Dortmund: Synergen Verlag.

NAGL Manfred (1972), Science Fiction in Deutschland, Tübingen: Tübinger Vereinigung für Volkskunde.

NiETZSCHE Friedrich (1994), Also sprach Zarathustra, Stuttgart: Philipp Reclam jun.

Novian Michael (2013), Von Ariern und Aliens. Völkische Weltanschauung in der Science-FictionLiteratur vor dem Zweiten Weltkrieg, Marburg: Tectum Verlag.

Peters Michael (o. J.), „Der Alldeutsche Verband“, Historisches Lexikon Bayerns, online unter < www.historisches-lexikon-bayerns.de/Lexikon/Alldeutscher_Verband_(ADV)>.

SAPRA Nessun (2005), Lexikon der deutschen Science Fiction und Fantasy 1870-1918, (mit einem Vorwort von Klaus Geus), Oberhaid: Utopica.

SAPRA Nessun (2007), Lexikon der deutschen Science Fiction und Fantasy 1919-1932 (mit einem Vorwort von Klaus Geus), Oberhaid: Utopica.

SCHWONKE Martin (1957), Vom Staatsroman zur Science Fiction, Stuttgart: Ferdinand Enke Verlag.

SEESTERN (= Ferdinand GRAUTOFF) (1905), 1906. Zusammenbruch der alten Welt, Leipzig:

Dieterich'sche Verlagsanstalt.

SPENGLER Oswald (1931), Der Mensch und die Technik, München: C. H. Beck.

SPENGLER Oswald (1981), Der Untergang des Abendlandes. Umrisse einer Morphologie der Weltgeschichte, München: Verlag C. H. Beck.

SÜNNER Rüdiger (1999), Schwarze Sonne. Entfesselung und Missbrauch der Mythen im Nationalsozialismus und rechter Esoterik, Freiburg, Basel, Wien: Herder Verlag.

Suvin Darko (1979), Poetik der Science Fiction. Zur Theorie und Geschichte einer literarischen Gattung, Frankfurt am Main: Suhrkamp Verlag.

THIELER Edith (1932), Max Haushofer. Literaturhistorische Untersuchungen über sein Leben und seine Dichtungen mit Herausstellung seines Zentralproblems, Königsberg: Buchdruckerei P. Escher.

Tzschaschel Roy (1989): „Druso“, F. Rollensteiner (Hrsg.), Werkführer durch die utopischphantastische Literatur, Meitingen: Corian Verlag. 
WEBER Heiko \& BREIDBACH Olaf (2006), „Der Deutsche Monistenbund 1906 bis 1933“, A. E. Lenz \& V. Mueller (Hrsgg.), Darwin, Haeckel und die Folgen. Monismus in Vergangenheit und Gegenwart, Neustadt am Rübenberge: Angelika Lenz Verlag, 157-206.

WEGENER Franz (2001), Das atlandische Weltbild. Nationalsozialismus und Neue Rechte auf der Suche nach der versunkenen Atlantis, Gladbeck: KFVR.

\section{NOTES}

1. Vgl. zur Begriffsabgrenzung von Science Fiction und Utopie: Jehmlich (1980).

2. Henning Franke stellt in Bezug auf die politisch-militärischen Science Fiction-Romane, die eine breite Überschneidung zur völkischen Science Fiction aufweisen, fest, dass die Auflagehöhe bis zu 200000 Exemplaren ging (vgl. Franke, 1985: 9).

3. Neben den beiden zitierten Studien liegen in deutscher Sprache nur noch Monographien von Henning Franke (1985), Roland Innerhofer (1996) und Michael Novian (2013) sowie einzelne Aufsätze zu diesem Themenkomplex vor.

4. Anfang der 2000er Jahre sorgte der rechtsradikale Aktivist und Politiker Christian Worch (Partei Die Rechte) in Deutschland für Aufsehen, weil er eine utopische SF-Trilogie unter dem Arbeitstitel Shayin verfasst hatte. Im Jahr 2008 erschien darüber hinaus - begleitet von kritischer Berichterstattung in den bundesdeutschen Feuilletons - die Romanreihe Stahlfront, von der seit 2009 mehrere Romane wegen Glorifizierung des Nationalsozialismus auf dem Index für jugendgefährdende Schriften zu finden sind.

5. Die Erstauflage betrug 1000 Exemplare (Franke, 1985: 330).

6. Siehe Hinweis bei Jokan (2016).

7. Vgl. Belleville Verlagshomepage <www.belleville-verlag.de/scripts/buch.php?ID=505> (20. Juni 2017).

8. Eine mutmaßlich gekürzte Fassung des Romans erschien in dem amerikanischen SF-Magazin Wonder Stories.

9. Druso (übersetzt von G. H. Gallet), Paris: Librairie Hachette, 1960 (es handelt sich auch hier um eine leicht gekürzte Fassung).

10. Vgl. zum Monistenbund u. a.: Weber \& Breidbach (2006: 157-206).

11. Vgl. (Thieler, 1932).

12. Vgl. Peters (o. J.).

13. Vgl. z. B. Tzschaschel (1989), Münch (2005).

14. Zur Instrumentalisierung des Atlantismythos vgl. auch: Sünner (1999: 44-53).

\section{RÉSUMÉS}

Der Beitrag thematisiert - basierend auf der Darstellung ausgewählter Beispiele von völkischen Zukunftsromanen aus dem deutschen Kaiserreich und der Weimarer Republik - inwiefern völkische Utopien durch Trivialliteratur verbreitet und hoffähig gemacht wurden. Die dargestellten Beispiele decken unterschiedliche Untergattungen des Genres ab und vermitteln einen Eindruck davon, wie bereits lange vor 1933 rassistische, chauvinistische und antisemitische Politik in der Belletristik verhandelt wurde. 
Cette contribution étudie des romans de science-fiction nationalistes et racistes qui sont parus à l'époque du Reich fondé en 1871 et du national-socialisme. Elle montre comment des utopies nationalistes et racistes ont été popularisées par ce type de paralittérature. Les exemples ici analysée correspondent à différentes sous-catégories du genre et permettent de comprendre comment une politique raciste, chauvine, antisémite a été diffusée bien avant 1933 dans la sphère littéraire.

Based on select examples of völkisch futuristic novels from the German Empire and the Weimar Republic this contribution explores the question to what extent völkisch utopias gained circulation and social acceptance owing to popular literature. Those examples represent various sub-genres and give an impression of how racist, chauvinistic and antisemitic policies were already the subject of literary fiction long before 1933.

\section{INDEX}

Mots-clés : science-fiction, völkisch, nationalisme, roman d'anticipation, Allemagne 1890-1933, Max Haushofer, Beowulf (= Maximilian Kern), Friedrich Freksa

Schlüsselwörter : Science Fiction, völkisch, Zukunftsroman, Deutschland 1890-1933, Max Haushofer, Beowulf (= Maximilian Kern), Friedrich Freksa

Keywords : science fiction, völkisch, nationalism, Germany 1890-1933, futuristic novel, Max Haushofer, Beowulf (= Maximilian Kern), Friedrich Freksa

\section{AUTEUR}

\section{MAURICE SCHUHMANN}

Freie Universität Berlin, Universität Hamburg 